\title{
Video Sequence Analysis for On-Table Tennis Player Ranking and Analysis
}

Xiaoni Wei, Hechi University, China*

\begin{abstract}
With the rapidly development of the scientific research in the field of sports, big data analytics and information science are used to carry out technical and tactical statistical analyses of competition or training videos. Table tennis is a skill-oriented sport. The technique and tactics in table tennis are the core factors to win the game. With the endlessly emerging innovative playing techniques and tactics, the players have their own competition styles. According to the competition events among athletes, the athletes' competition relationship network is constructed, and the players' rankings are established. The ranking can be used to help table tennis players improve daily training and understand their ability. In this paper, the table tennis players' rankings are established from their competition videos and their prestige scores in the table tennis players' competition relationship network.
\end{abstract}

\section{KEYWORDS}

Competition Relationship Network, Players' Ranking, Video Sequence Analysis

\section{INTRODUCTION}

With the rapid development of information technology, artificial intelligence (AI) (Dwivedi et al. 2019; Vaishya et al. 2020), Internet of Things (IoT) and cloud computing (Jahantigh et al. 2019; Haji et al. 2020) have been widely used in social development. Relevant scientific research in the field of sports is also developing rapidly (Козина et al. 2017; Matic et al. 2020). The big data analysis has been widely used to carry out technical and tactical statistical analysis for competition videos. As a skill oriented net competition project, the proper skills and tactics are the core of table tennis for the victory. With the development of table tennis sports, various innovative techniques and tactics emerge one after another. Athletes in various countries have their own competition style. Scientific statistical analysis of various techniques and tactics can help athletes win the competition (Bańkosz \& Winiarski 2020; Ma 2020).

The study of table tennis techniques and tactics is the main theme of table tennis sports (Wang et al. 2019; Huang et al. 2021). Among them, scientific research scholars mainly use two statistical methods: manual table statistics and computer statistics for technical and tactical analysis. The initial method of technical and tactical statistical analysis is manual table statistics, which is a way for researchers to design index system according to research needs and use tables for statistical analysis with reference to statistical contents. Manual statistics has the advantages, including convenient material acquisition, less investment, large audience and convenient application. However, it also 
has its disadvantages. The statistical speed is slow and the process is cumbersome. The amount of statistical information is huge and needs the cooperation of many people. There are human factors in the statistical results, which is easy to lead to differences in statistical data. The post-processing of statistical data costs lots of time and is difficult to reuse.

Computer statistics (Ceri 2018; Andersson \& Kroisandt 2018) is an intelligent processing method for video analysis based on the statistical purpose and statistical demand through machine learning and video analysis. Computer-aided system (Wang \& Jiang 2021; Zhang \& Liu 2021) has become a development trend of technical and tactical analysis in athletic sports, such as table tennis sports. A well-designed technical and tactical analysis and statistics system can improve statistical efficiency and video utilization, greatly save time, reduce the cost, avoid subjective assumptions, overcome most defects of manual statistics, and provide relevant data for coaches and athletes.

The intelligent technical and tactical statistical analysis of video data combined with machine learning has been widely applied in athletic sports training and competition (Herold et al. 2019; Wenninger et al.2020). Table tennis statistical index refers to the statistical content designed according to the research purpose to cover the utilization of techniques and tactics of table tennis players in the competition. The difference of statistical needs, statistical means and statistical objects will lead to the difference of statistical objectives. The collection and statistics of table tennis technical and tactical data need experienced researchers or researchers with a deep understanding of table tennis technology and skills. By combining with table tennis technical and tactical analysis theory, the coacher can design statistical indicators to help athletes grasp the correct technical and tactical characteristics.

There are many factors to win table tennis competition. Among them, the control of landing point is an indispensable point of scoring. Between the rounds of each ball, the landing point of the previous ball will have a relevant impact on the threat of the other party's return ball. A good hitting landing point is very important in the table tennis competition.

Statistical index is the embodiment of the concretization of statistical content and the core issue of technical and tactical analysis. It reflects the concept and specific value characteristics of the overall phenomenon. With the help of the analysis of index system, it can effectively reflect the essential characteristics and laws of technical and tactical in the competition to construct statistical index system for table tennis techniques and tactics. The statistical index system should follow the scientific principle, comprehensiveness principle, and measurement principle.

The scientific principle refers to that the design of technical and tactical video statistical indicators should aim at the special characteristics of table tennis, take scientific thought as the guidance, be able to establish a real, accurate, objective and scientific index system, take the statistical purpose and demand as the standard, take the corresponding discrimination standard as the evaluation scale, and take the expert consultation and questionnaire results as the basis with clear content and mutual independence to build a scientific and feasible index system.

The comprehensiveness principle refers to that the statistical index system should fully cover all statistical indicators to make it be comprehensive. According to the different statistical objects and statistical needs, the statistical indicators adopted by researchers are also different. Through consulting the literature, it can be seen that more researchers focus on the statistics of technology and tactics, and adopt the three-stage index or four-stage index evaluation method. The landing point in table tennis competition is not only the key point of scoring, but also a measurable index. Simply analyzing the use of technologies and tactics cannot fully explain the situation of the game.

The measurement principle requires the selected index must be objective and qualitative to avoid the uncertainty of statistical results. The statistical index data is an intuitive embodiment of the situation of athletes in the sports. The index content is the key factor to build a table tennis technical and tactical video statistical system. The meaning of the index needs to be clear. The data should be easy to collect.

The primary indexes are applied to the actual measurement of the sports team, and the index contents are revised according to the modification opinions. Through the questionnaire survey to test 
the rationality of the index system, we can screen the failed indexes and improve the contents covered by the index system. The questionnaire contains three criteria level indicators of tactics, technology and landing point. It can more accurately express expert opinions and opinions, reflect the results of the questionnaire survey, achieve the purpose of the questionnaire survey, and is conducive to the accuracy and reliability of the whole questionnaire survey results. This paper considers tactical indicators, technical indicators, landing point indicators and gain and loss indicators by combining with the special characteristics of table tennis and taking the video technical and tactical statistical indicators of table tennis competition as the starting point. Then, the index system is constructed according to technical and tactical video statistics for table tennis competition. The establishment of the index system lays a solid foundation for the construction of table tennis technical and tactical video statistics system to ensure the smooth development of the follow-up statistical work. Then, the results from index system are further used to analyze the ranking of the table tennis players.

Based on the node centrality research of complex network (Saleh et al. 2018; Baravalle et al. 2019), this paper proposes athlete's ability ranking through the analytical results of the training and competition videos of table tennis players to show their potential competition ability. Firstly, we collect all the table tennis training and competition videos. The analysis results are converted as the network relationship. The times between athletes in training or competition are used as the edge weight. Then, the athlete ranking is proposed to quantify the importance of the athletes by using the prestige score of the athletes. Finally, the athletes are ranked through the experimental data, and then compared with the ranking given by coaches or experts to verify the effectiveness of the proposed ranking method.

\section{COMPUTER-AIDED SYSTEM TO ASSIST TABLE TENNIS PLAYER TRAINING THROUGH COMPETITION VIDEO AND DATA ANALYSIS}

A social system often has complex characteristics. The complexity is generally shown at that the human behavior often follows complex dynamic patterns, such as behavior deletion in the establishment of electronic communication rules. At the same time, the complexity is also reflected in the overall level. For example, when the social system is represented in the mathematical form of graph or network, the node represents the individual, and the edge represents the interactive relationship between individuals in social activities. In most cases, social networks are scale-free from the perspective of topology and global.

In recent years, complex networks have become an important approach to study social system problems and a hot topic in the research of interdisciplinary (Ding et al. 2019; Cherifi et al. 2019). Current social activities release a large amount of data to describe human activities and their interaction. With the development of data science and network science, it has become an important task of artificial intelligence research by using big data technology to analyze the interdependence between massive data, mine the internal laws of data and reflect the relationship of data objects. For the current social system, a new trend is to analyze the macro statistical characteristics of social interaction system composed of a large number of individuals other than the behavior of a single individual. The aim is to find and apply the laws. From this aspect, large-scale data about sports events are processed by complex network, such as football, rugby, baseball, and basketball. In this paper, the complex network is adopted to analyze the table tennis training or competition data.

The table tennis players are used as the network topology nodes and the competition events between players are used as the edge relationship to construct a network topology which is used to describe and mine the big data in the table tennis events.

Obviously, it is a very natural expression in the social system that the competition can be regarded as the basic contact between two players. When a table tennis player $i$ wins the player $j$ in the competition, there is a contiguous relationship between these two players to form a peerto-peer network topology. The edge weights are adopted to further mine the topological relationship. The edge weight is equal to the number of competition events between two table tennis players. 
Then, a non-peer-to-peer network topology is constructed. The constructed network with weights has a high degree of freedom, which can allow the display of table tennis competition relationship networks in various time periods, levels and types. Generally speaking, the network aggregated from a large amount of competition or training data has similar network topology complexity characteristics with the social network. The classical method to measure complex networks is to calculate the probability density distribution of node degree, and the result follows a significant power-law distribution. In the athlete competition relationship network, it means that most athletes only participate in part of the competition or training. A few top athletes will participate in most competitions and beat strong opponents.

This paper aims to rank athletes on the basis of complex network topology. In the network, each athlete has a prestige score to evaluate their ranking in the table tennis sports. Let us assume that the prestige is a variable in the edge weighted network and athletes can improve the ranking by constantly obtaining other people's prestige scores. The process can be solved by establishing the following equation to obtain the system solution:

$p_{i}=(1-q) \sum_{j} p_{j} \frac{w_{i, j}}{k_{j}}+\frac{q}{N}+\frac{1-q}{N} \sum_{j} p_{j} \delta\left(k_{j}\right)$

In the Equation (1), $i$ or $j$ represents a node in the complex network, $p_{i}$ satisfies $\sum_{j} p_{j}=1$, $N$ represents the number of table tennis players, $k_{j}$ represents the weight of node $j$. The $p_{j}$ can be regarded as the prestige score function, which represents the ranking of player $j$ in all table tennis players. The $q \in[0,1]$ is a control parameter to balance the contribution of each node to the complex network. The term, $(1-q) \sum_{j} p_{j} \frac{w_{i, j}}{k_{j}}$ represents the score proportion of node $i$ during prestige diffusion process. It means that each node redistributes its personal credit to its neighbor nodes depends on the edge weight between two nodes. The $\frac{q}{N}$ reflects that the prestige of table tennis players is evenly distributed to all nodes, and then each player in the network receives a fixed and equal number of credit prestige score. The term, $\frac{1-q}{N} \sum_{j} p_{j} \delta\left(k_{j}\right)$, is equal to 0 when $q=1$.

For a general network topology, the Equation (1) is difficult to be obtained by numerical calculation because only the stable value of Equation (1) is the result of infinite recursive operations. This paper initializes $p_{i}=\frac{1}{N}$ to iteratively calculate Equation (1) until it converges to the stable value with a priori fixed accuracy $a$.

In the athlete competition relationship network, the simplest network topology is the result extracted from the data of a simple team training. For this kind of network, the solution of Equation (1) can be obtained by analyzing the related data. In individual championships, the competition relationship network is organized as a hierarchical structure, such as a binary tree. Let $r$ represent the depth of table tennis players, then $N=2^{r}$. The Equation (1) can be simplified as a function about the number of competitions $r$ with promoted athletes. Then, the Equation (1) is rewritten as follows:

$$
p_{r}=p_{0}+(1-q) \sum_{u=1}^{r}\left(p_{u}-1\right)
$$


In the Equation (2), $p_{0}=\frac{1-q}{N} p_{1}+\frac{q}{N}$ and $0 \leq t \leq 1$. The score function $p_{r}$ is the sum of two parts. The first term, $p_{0}$, represents the average contribution of all qualified players. The second term, $(1-q) \sum_{u=1}^{r}\left(p_{u}-1\right)$, represents the score of players' promotion and winning competition. The following iterative relation holds:

$p_{r}=(2-q) p_{r-1}=\ldots=(2-q)^{r} p_{0}$

From the Equation (3), it can be found that the Equation (3) relies on a constant value and satisfies the following normalization condition:

$$
\sum_{r=0}^{l} n_{r} p_{r}=1
$$

In the Equation (4), $n_{r}$ represents the number of athletes who win $r$ competitions. For individual championship, $n_{r}=2^{l-r-1}$ holds $(0<r<1)$. Through $\sum_{r=0}^{u} x^{r}=\frac{1-x^{u+1}}{1-x}$, the initialized value of $p_{0}$ can be obtained by the following equation:

$p_{0}=\frac{q}{2^{l}+(2-q)^{l}(q-1)}$

By combining the Equation (3) and (5), we can obtain the following equation:

$$
p_{r}=\frac{q(2-q)^{r}}{2^{l}+(2-q)^{l}(q-1)}
$$

It should be noted that if $q=1$, the Equation (6) can be simplified to $p_{r}=2^{-l}$, which shows that prestige is homogeneous and evenly distributed in the network without diffusion. On the contrary, if $q=0$, the Equation (6) is transformed as the following equation:

$$
p_{r}=\frac{2^{r}}{2^{l}(l+2)}
$$

\section{EXPERIMENTS AND SIMULATIONS}

In this section, we collect the daily training and competition videos of table tennis players from a university. We collect 50 college students' daily training and competition data. The nodes in the complex network depict the information of the table tennis players in daily training and competition videos. The following figure illustrates the relationship between $p_{r}$ and $r$. 
Figure 1. The distribution of $p_{r}$ with different parameter $q$

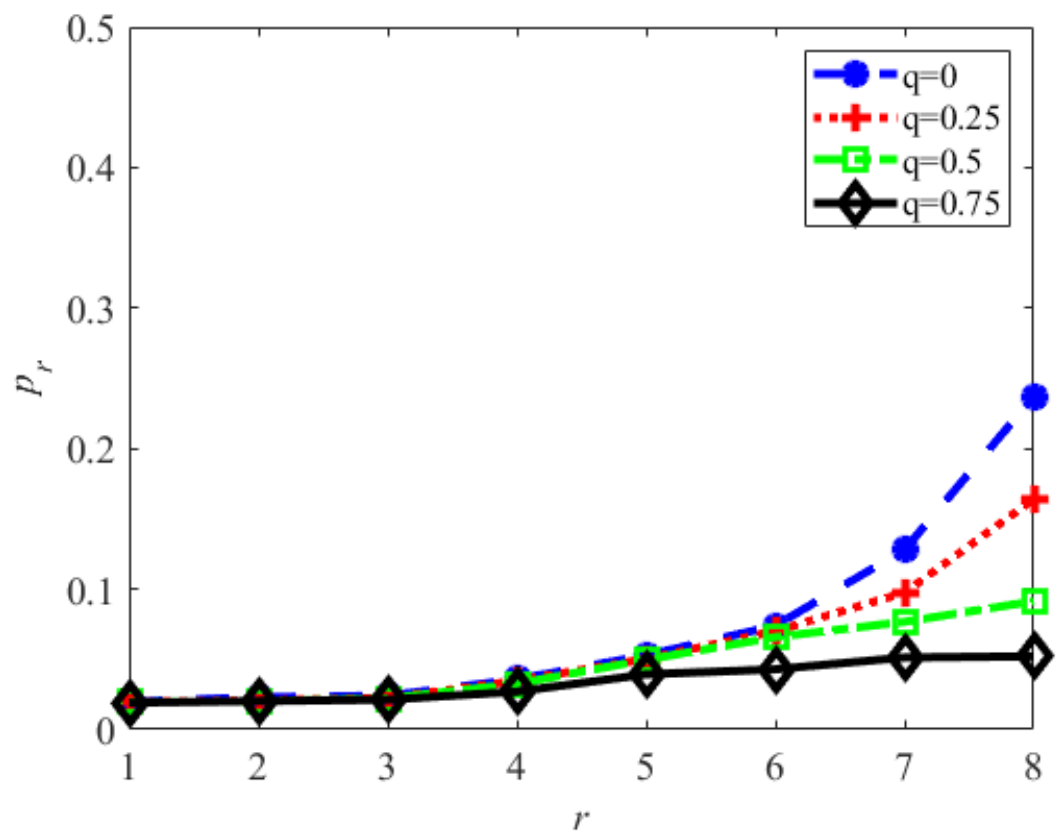

In general, a low parameter $q$ makes the players in last round of competition have two orders of magnitude higher scores than the opponent in the first round. The best player should be in the form of exponential function of the number round in competition, $l$. In our experiment, the parameter $q$ is set as 0.25 . Table 1 shows the analysis of the athlete competition relationship network constructed by the table tennis training and competition data, including the prestige ranking of the top 10 athletes. In order to protect the privacy of the college student players, the Table 1 only lists the surname of the players.

Then, the results in Table 1 are compared with the evaluation of coaches, which shows the prestige scoring method based on complex network proposed in this paper can effectively rank athletes in

Table 1. The top 10 prestige ranking of table tennis players in universities

\begin{tabular}{|l|l|l|}
\hline \multicolumn{1}{|c|}{ Order } & \multicolumn{1}{c|}{ Name } \\
\hline 1 & Zhang $* *$ & PKU \\
\hline 2 & Liu $* *$ & BJUT \\
\hline 3 & Wang $* *$ & RUC \\
\hline 4 & Qian $* *$ & BFSU \\
\hline 5 & Chen $* *$ & THU \\
\hline 6 & Cheng $* *$ & BNU \\
\hline 7 & Xu $* *$ & CUC \\
\hline 8 & Zheng $* *$ & USTB \\
\hline 9 & Yang $* *$ & CUFE \\
\hline 10 & Hu $* *$ & BJRU \\
\hline
\end{tabular}




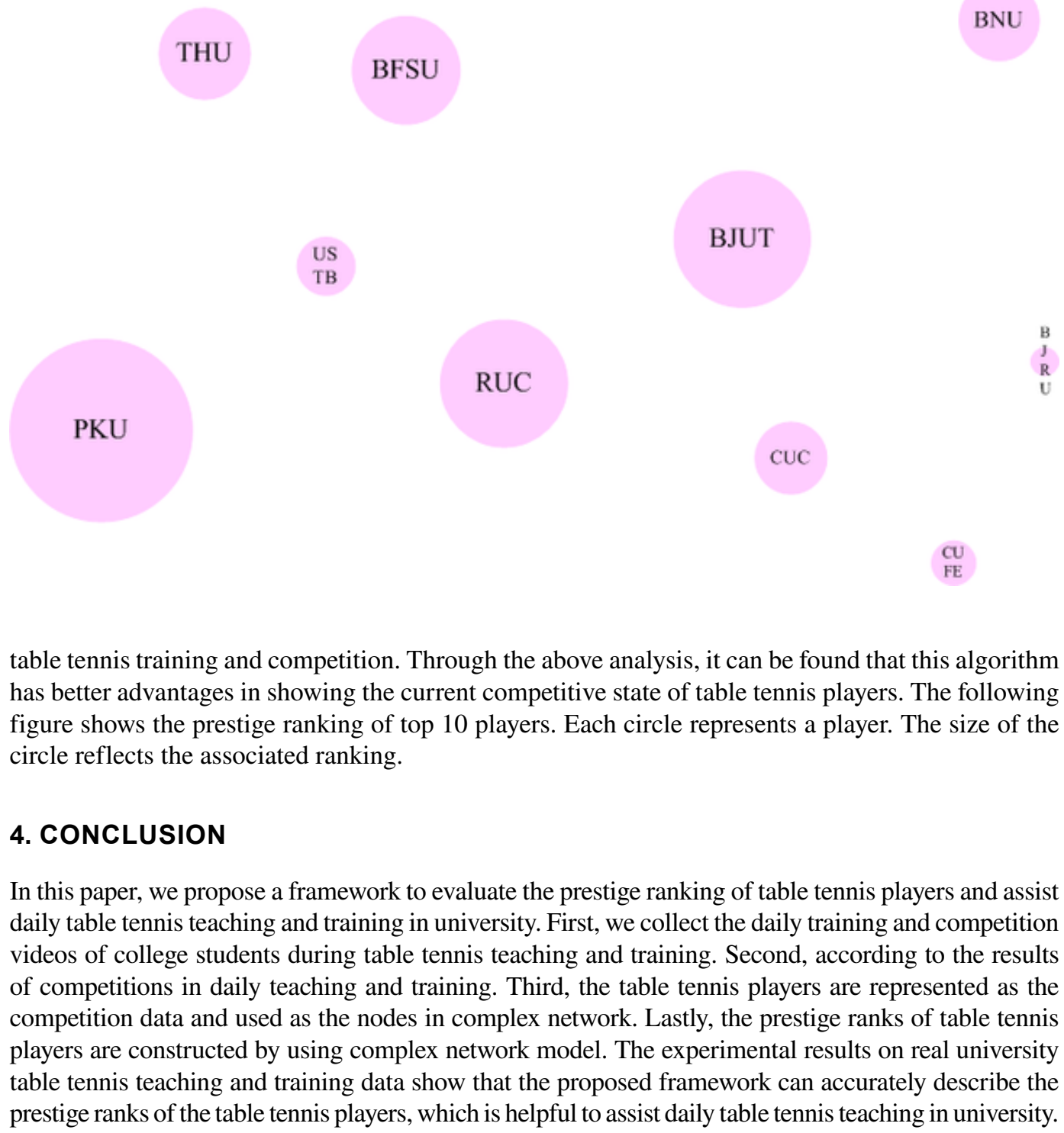

\section{ACKNOWLEDGMENT}

This research received no specific grant from any funding agency in the public, commercial, or notfor-profit sectors. 


\section{REFERENCES}

Козина, Ж. Л., Репко, Е. А., Козин, С. В., Цеслицка, М. З., \& Мушкета, Р. К. (2017). Application of system analysis in scientific research in the field of sports. Health, Sport, Rehabilitation, 3(4), 3-12.

Andersson, C., \& Kroisandt, G. (2018, August). Key components of an E-Learning module in statistics for computer science students. In 2018 13th International Conference on Computer Science \& Education (ICCSE) (pp. 1-4). IEEE.

Bańkosz, Z., \& Winiarski, S. (2020). Statistical parametric mapping reveals subtle gender differences in angular movements in table tennis topspin backhand. International Journal of Environmental Research and Public Health, 17(19), 6996. doi:10.3390/ijerph17196996 PMID:32987863

Baravalle, R., Guisande, N., Granado, M., Rosso, O. A., \& Montani, F. (2019). Characterization of visuomotor/ imaginary movements in EEG: An information theory and complex network approach. Frontiers in Physics, 7, 115.

Ceri, S. (2018). On the role of statistics in the era of big data: A computer science perspective. Statistics \& Probability Letters, 136, 68-72. doi:10.1016/j.spl.2018.02.019

Cherifi, H., Palla, G., Szymanski, B. K., \& Lu, X. (2019). On community structure in complex networks: Challenges and opportunities. Applied Network Science, 4(1), 1-35.

Ding, R., Ujang, N., Hamid, H. B., Abd Manan, M. S., Li, R., Albadareen, S. S. M., \& Wu, J. et al. (2019). Application of complex networks theory in urban traffic network researches. Networks and Spatial Economics, 19(4), 1281-1317.

Dwivedi, Y. K., Hughes, L., Ismagilova, E., Aarts, G., Coombs, C., Crick, T., \& Williams, M. D. et al. (2019). Artificial Intelligence (AI): Multidisciplinary perspectives on emerging challenges, opportunities, and agenda for research, practice and policy. International Journal of Information Management, 101994.

Haji, L. M., Ahmad, O. M., Zeebaree, S. R., Dino, H. I., Zebari, R. R., \& Shukur, H. M. (2020). Impact of cloud computing and internet of things on the future internet. Technology Reports of Kansai University, 62(5), 2179-2190.

Herold, M., Goes, F., Nopp, S., Bauer, P., Thompson, C., \& Meyer, T. (2019). Machine learning in men's professional football: Current applications and future directions for improving attacking play. International Journal of Sports Science \& Coaching, 14(6), 798-817.

Huang, W., Lu, M., Zeng, Y., Hu, M., \& Xiao, Y. (2021). Technical and tactical diagnosis model of table tennis matches based on BP neural network. BMC Sports Science, Medicine and Rehabilitation, 13(1), 1-11. doi:10.1186/s13102-021-00283-3 PMID:34016177

Jahantigh, M. N., Rahmani, A. M., Navimirour, N. J., \& Rezaee, A. (2019). Integration of internet of things and cloud computing: A systematic survey. IET Communications, 14(2), 165-176. doi:10.1049/iet-com.2019.0537

Ma, H. (2020). Improvement of table tennis technology based on data mining in the environment of wireless sensor networks. International Journal of Distributed Sensor Networks, 16(10), 1550147720961343. doi: $10.1177 / 1550147720961343$

Matic, R. M., Popovic, S., Maksimovic, N., Bjelica, D., \& Vukovic, J. (2020). Attitudes of academic staff from different stages of their proficiency in research and teaching activities in sports sciences: A case study of the University of Montenegro. Sport Mont, 18(3), 25-30.

Saleh, M., Esa, Y., \& Mohamed, A. (2018). Applications of complex network analysis in electric power systems. Energies, 11(6), 1381.

Vaishya, R., Javaid, M., Khan, I. H., \& Haleem, A. (2020). Artificial Intelligence (AI) applications for COVID-19 pandemic. Diabetes \& Metabolic Syndrome, 14(4), 337-339. doi:10.1016/j.dsx.2020.04.012 PMID:32305024

Wang, J., Zhao, K., Deng, D., Cao, A., Xie, X., Zhou, Z., Zhang, H., \& Wu, Y. (2019). Tac-simur: Tactic-based simulative visual analytics of table tennis. IEEE Transactions on Visualization and Computer Graphics, 26(1), 407-417. doi:10.1109/TVCG.2019.2934630 PMID:31442999 
Wang, X., \& Jiang, C. (2021). Computer-aided physical training sports injury risk simulation based on embedded image system. Microprocessors and Microsystems, 83, 103975.

Wenninger, S., Link, D., \& Lames, M. (2020). Performance of machine learning models in application to beach volleyball data. International Journal of Computer Science in Sport, 19(1).

Zhang, S., \& Liu, M. (2021). Computer aided management system of sports horse registration based on distributed storage system and deep fusion learning. Microprocessors and Microsystems, 104120. 